\title{
High-efficiency fluorescent organic light-emitting device with double emissive materials
}

\author{
Huishan Yang ${ }^{\mathrm{a}}$, Lishuang $\mathrm{Wu}^{\mathrm{b}}$, Zhiwei $\mathrm{Wu}^{\mathrm{c}}$ \\ College of Physics and Information Engineering, Quanzhou Normal University, Quanzhou 362000, \\ People's Republic of China \\ a email:yanghuishan1697@163.com, bemail:lishuangw@126.com; c wzwqq@126.com
}

Keywords: White organic light-emitting devices; Luminance; Current efficiency; Power efficiency Abstract. A fluorescent non-doped white organic light-emitting device (WOLED) with double emissive layers was fabricated. The yellow and blue dyes, 4- (dicyanomethylene) -2-t-butyle-6-(1, 1, 7, 7- tetramethyljulolidyl-9-enyl)-4H-pyran (DCJTB) and 9, 10-di (2-naphthyl) anthracene (ADN), can be realized by blending complementary colors. The maximum current efficiency and power efficiency of the WOLED are $7.61 \mathrm{~cd} / \mathrm{A}$ at $9 \mathrm{~V}$ and $2.11 \mathrm{~m} / \mathrm{W}$ at $8 \mathrm{~V}$, with the maximum brightness of 15840 $\mathrm{cd} / \mathrm{m} 2$ at $12 \mathrm{~V}$. The Commission International deL'E'clairage coordinates change slightly from $(0.5154,0.4754)$ to $(0.4602,0.4427)$, as the applied voltage increases from $4 \mathrm{~V}$ to $12 \mathrm{~V}$. The high efficiencies can be attributed to the decrease concentration quenching of fluorescence dye.

\section{Introduction}

Since Tang and VanSlyke1 from Kodak introduced efficient organic light-emitting devices ( OLEDs ), they have been widely investigated because of their possible application in flat panel displays and lighting applications [1-4]. Through these research efforts, many achievements have been made. Satisfactory white organic light-emitting diodes (WOLEDs) are always constructed on a multilayer device structure with two (green blue/orange red or blue/yellow) to three (red, green, and blue) light-emitting components [5-24]. Among these color components of WOLEDs, many blue, most of yellow, and nearly all orange to red dye suffer from a common problem, namely the concentration quenching of fluorescence in solid state. Consequently, the dopant light-emitter of guest-host system becomes a universal method for solving the quenching problem, orange to red dye [25]. Doping process is one of the key methods, has been generally used to enhance the characteristics of the OLEDs. However, in practical OLED manufacture, the doping process is hard task to handle. To overcome these problems, no-doping technique has been reported, and several attempts have been made in making some head way in achieving enhanced characteristics in the OLEDs [26-27]. In this letter, the fabrication of multilayer WOLEDs did not involve the hard-to-control doping process. Sub -monolayer DCJTB act as red emitting layer, ADN act as blue emitting layer, the effect of ADN emission on the WOLED is demonstrated. The simple device structures and excellent reproducibility make them well suited to low-cost lighting applications and beneficial to the industrialization.

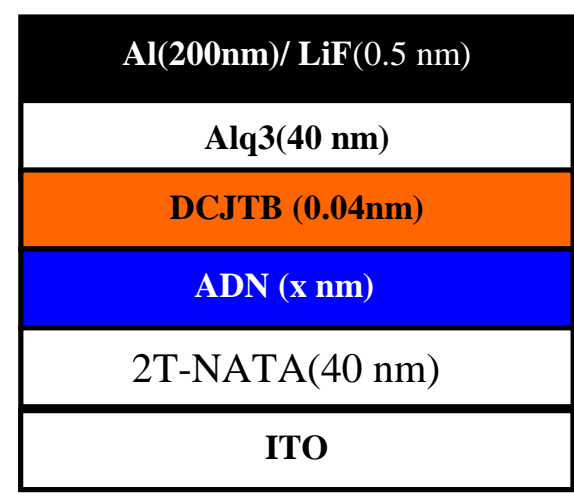

Fig. 1 The device structure of the OLEDs 


\section{Experimental}

Fig. 1 shows the chemical structure of organic materials and the device structure of the OLEDs. The devices are prepared in a vacuum chamber at a pressure of $4 * 10^{-4}$ pa by thermal evaporation onto a cleaned indium tin oxide (ITO) coated glass substrate. The multilayer devices fabricated have the following structure: ITO/ 2T-NATA(40 nm)/ ADN (x nm)/ DCJTB (0.04 nm)/Alq3(40 nm)/LiF (0.5 $\mathrm{nm}$ ) /Al, where $\mathrm{x}=15,20,25$ and 30, called device A-D, respectively. A bilayer of lithiumfluoride $(\mathrm{LiF})$ and aluminium $(\mathrm{Al})$ were used for the cathode. The thickness of layers was controlled by quartz crystal monitor. The EL spectra and CIE coordination of the devices were measured by PR655 Spectra Scan spectrophotometer and the current-voltage-brightness characteristics were simultaneously measured by a voltage-current source (Keithley 2400) All the measurements were carried out at room temperature under ambient atmosphere.

\section{Results and discussion}

In general, the EL spectra and the CIE coordinates of the white light-emitting device are strongly influenced by the concentration of the dyes as well as the thickness of each emissive layer. First, we consider the most suitable concentration for the different dyes. As shown in Fig.2, the normalized EL spectra of the devices shows two main emission peaks at $440 \mathrm{~nm}$ and $554 \mathrm{~nm}$ originating from ADN and DCJTB, respectively. With the thickness of AND increased, the yellow emission is enhanced. The yellow emission stronger compared with that blue emission, this observation is attributed to the fact that more excitons are captured by DCJTB. With the applied voltage increased, the blue emission enhances. When the thickness of blue emissive layer increases, the number of the electrons reaching the blue emissive layer will reduce in contrast to the increasing electrons staying at the yellow emissive layer, because of the no good hole-transporting property of AND. In sequence, the exciton formation probability in the blue emissive layer will reduce accordingly. As a consequence, the blue emission will reduce with the yellow emission increasing. The CIE coordinates of devices A-D at different voltage shown in Fig 3. We can see device A and B appear green white, and devices C-D shows yellow white.
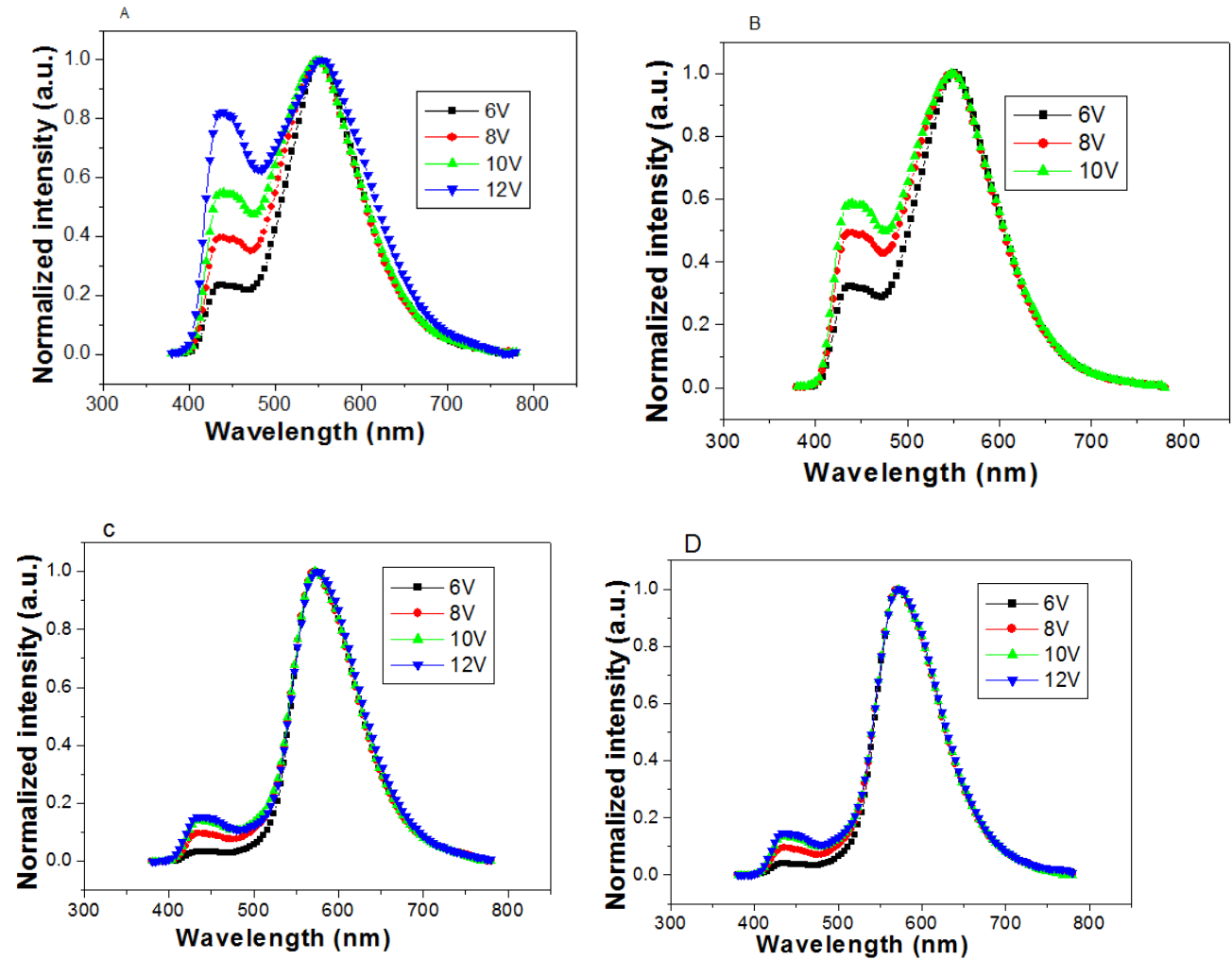

Fig. 2. Normalized EL intensity of the different devices A-D at different voltage 


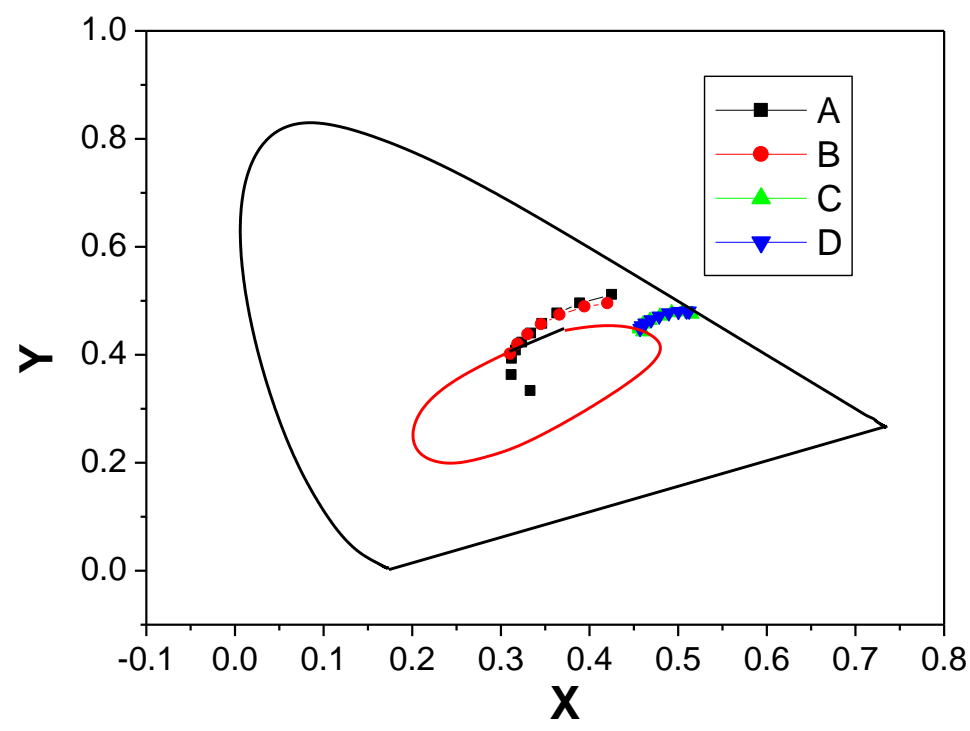

Fig 3. CIE coordinates of devices A-D at different voltage

Fig.4 shows the current density versus voltage $(\mathrm{J}-\mathrm{V})$ characteristics of the devices. The luminance-voltage characteristics of the devices are depicted in Fig.5. For example, the brightness of devices A-D is $7669 \mathrm{~cd} / \mathrm{m} 2$ at a given voltage of $12 \mathrm{~V}, 1598 \mathrm{~cd} / \mathrm{m} 2$ at a given voltage of $10 \mathrm{~V}, 15840$ $\mathrm{cd} / \mathrm{m} 2$ at a given voltage of $12 \mathrm{~V}$ and $5276 \mathrm{~cd} / \mathrm{m} 2$ at a given voltage of $14 \mathrm{~V}$, respectively.

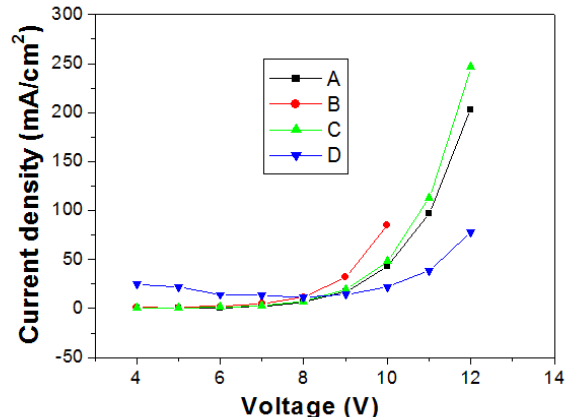

Fig 4. The current density versus voltage characteristics of the devices A-D

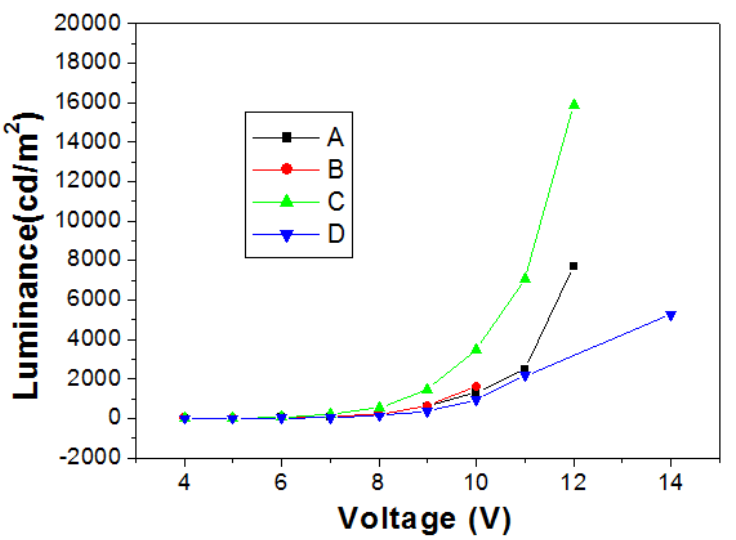

Fig 5. The luminance- voltage characteristics of devices A-D

Fig.6 shows the dependence of current efficiency on voltage for the devices. The maximum efficiency of devices A-D is $3.70 \mathrm{~cd} / \mathrm{A}$ at $7 \mathrm{~V}, 1.96 \mathrm{~cd} / \mathrm{A}$ at $8 \mathrm{~V}, 7.61 \mathrm{~cd} / \mathrm{A}$ at $9 \mathrm{~V}$ and $6.76 \mathrm{~cd} / \mathrm{A}$ at $14 \mathrm{~V}$, respectively. The device $C$ exhibits the best performance, followed by the device $\mathrm{D}$, the device $\mathrm{A}$, and the device B. The enhanced EL efficiency can be attributed to appropriate thickness of AND, the stronger accumulation of electrons and holes in the yellow emitting layer. The difference thickness of AND, resulting the excitons formation probability. The current efficiency is comparatively depends on the thickness of AND. Our results indicate that for a given device structure, the proper the thickness of AND can enhances the performance of organic emitting device. 

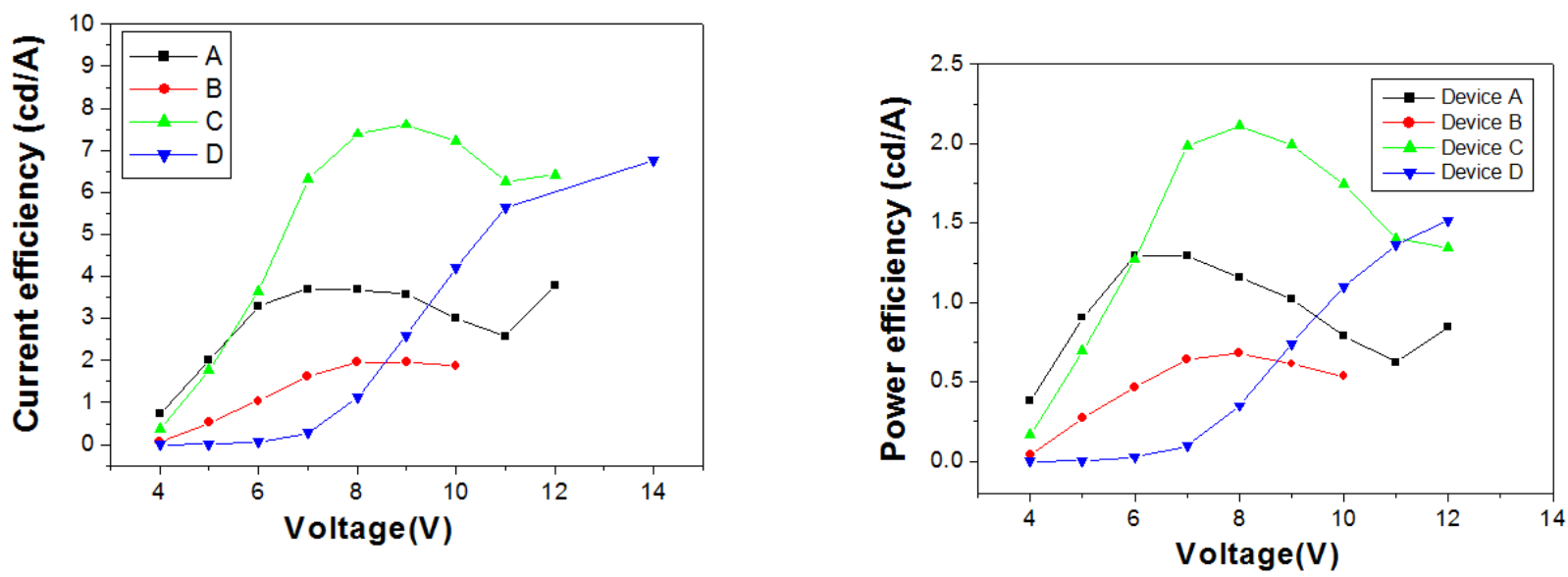

Fig 6. The current efficiency- voltage characteristics and power efficiency-voltage of devices A-D

\section{Summary}

In this letter, the fabrication of multilayer OLEDs did not involve the hard-to-control doping process. Sub -monolayer DCJTB act as yellow emitting layer. The simple device structures and excellent reproducibility make them well suited to low-cost lighting applications and beneficial to the industrialization. The yellow and blue dyes, DCJTB and ADN, can be realized by blending complementary colors. The maximum current efficiency and power efficiency of the WOLED are $7.61 \mathrm{~cd} / \mathrm{A}$ at $9 \mathrm{~V}$ and $2.11 \mathrm{~m} / \mathrm{W}$ at $8 \mathrm{~V}$, with the maximum brightness of $15840 \mathrm{~cd} / \mathrm{m} 2$ at $12 \mathrm{~V}$. The Commission International deL'E'clairage coordinates change slightly from $(0.5154,0.4754)$ to $(0.4602,0.4427)$, as the applied voltage increases from $4 \mathrm{~V}$ to $12 \mathrm{~V}$. The high efficiencies can be attributed to the decrease concentration quenching of fluorescence dye.

\section{Acknowledgements}

This work was supported by the major project of science and technology office of Fujian Province of China (No.2014H0042), the Natural Science Foundation of Fujian Province of China (No. 2015J01664), and the project of Science and Technology Research of Quanzhou, Fujian Provice of China (No.2013Z125,2014Z137).

\section{References}

[1] C. W. Tang, S. A. VanSlyke:Appl. Phys. Lett, Vol. 51 (1987), p 913

[2] Takayuki Chiba, Yong-Jin Pu, Ryoichi Miyazaki, Ken-ichi Nakayama, Hisahiro Sasabe, Junji Kido: Organic Electronics, Vol.12 (2011) , p710

[3] L. S. Hung, C. W. Tang, M. G. Mason: Appl. Phys. Lett, Vol. 70(1997),p152

[4] Dong-Hyun Lee, Jong Hyeok Park, Heeyeop Chae, Sung M. Cho: Organic Electronics, Vol. 10 (2009), p1345

[5] B. W. D’Andrade, S. R. Forrest: Adv.Mater, Vol. 16(2004), p1585

[6] J. Kido, M. Kimura, K. Nagai: Science, Vol. 267(1995), p1332

[7] R. S. Deshpande, V. Bulovic, S. R. Forrest :Appl.Phys.Lett, Vol. 75(1999),p888

[8] Z. Y. Xie, J. S. Huang, C. N. Li, S. Y. Liu, Y. Wang, Y. Q. Li, J. C. Shen :Appl.Phys.Lett, Vol. 74(1999),p641

[9] M. Strukelj, R. H. Jordan, A.Dodabalapur : J.Am.Chem.Soc, Vol. 118(1996),p1213 
[10] R. H. Jordan, A. Dodabalapur, M. Strukelj, T. M. Miller : Appl.Phys.Lett, Vol. 68(1996),p1192 [11] F. Steuber, J. Staudigel, M. Stossel, J. Simmerer, A. Winnacker, H. Spreitzer, F. Weisso“"rtel, J. Salbeck :Adv.Mater, Vol. 12(2000),p130

[12] S. Liu, J. Huang, Z. Xie, Y. Wang, B.Chen:Thin Solid Films, Vol. 363(2000),p294

[13] C. W. Ko, Y. T. Tao: Appl.Phys.Lett, Vol. 79(2001),p4234

[14] K. O. Cheon, J. Shinar: Appl.Phys.Lett, Vol. 81(2002),p1738

[15] Y.S.Hunag,J.H.Jou,W.K.Weng,J.M.Liu :Appl.Phys.Lett, Vol. 80(2002),p2782

[16]B.W.D’Andrade,M.E.Thompson,S.R.Forrest :Adv.Mater, Vol. 14(2002),p147

[17]C.H.Chuen,Y.T.Tao : Appl.Phys.Lett, Vol. 81(2002),p4499

[18]G.Li,J.Shinar : Appl. Phys. Lett, Vol. 83(2003),p5359

[19]X.H.Zhang,M.W.Liu,O.Y.Wong,C.S.Lee,H.L.Kwong,S.T.Lee,S.K.Wu:Chem.Phys.Lett, Vol. 369(2003),p478

[20] S.Tokito,T.Iijima,T.Tsuzuki,F.Sato: Appl.Phys.Lett, Vol. 83(2003),p2459

[21]T.-H.Liu,Y.-S.Wu,M.-T.Lee,H.-H.Chen,C.-H.Liao,C.H.Chen: Appl.Phys.Lett, Vol. 85(2004),p4304

[22]G.Cheng,Y.Zhao,Y.Zhang,S.Liu,F.He,H.Zhang,Y.Ma: Appl.Phys.Lett, Vol. 84(2004),p4457

[23]D.Qin,Y.Tao: Appl.Phys.Lett,Vol. 86(2005),p113507

[24]Y.Xu,J.Peng,Y.Mo,Q.Hou,Y.Cao:Appl.Phys.Lett, Vol. 86(2005),p163502

[25]C.-T.Chen: Chem.Mater, Vol. 16(2004),p4389

[26]T.Tsuji,S.Naka,H.Okada,H.Onnagawa:Appl.Phys.Lett, Vol. 81(2002),p3329

[27]W.F.Xie,Z.J.Wu,S.Y.Liu,S.T.Lee:J.Phys.D:Appl.Phys, Vol. 36(2003),p2331 